\title{
Dependence estimation and controlled CVaR portfolio optimization of a highly kurtotic Australian mining sample of stocks
}

\author{
$\underline{\text { J. Arreola }}^{a}$, D. Allen ${ }^{a}$ and R. Powell ${ }^{a}$ \\ ${ }^{\text {a }}$ School of Accounting, Finance and Economics, Edith Cowan University, Western Australia \\ Email: j.arreolahernandez.ecu.edu.au
}

\begin{abstract}
The drivers of mining stock prices are known to be several. Sharp spikes on the stocks return distribution have been linked to the presence of unusually high volatility signifying the presence of high levels of kurtosis. The accurate measurement of the stocks' underlying co-movements for more accurate CVaR portfolio optimization requires, therefore, the utilization of sophisticated and specific-specialized techniques which could adequately capture and model these characteristics. Here this issue is addressed by applying statistical-graphical models for dependence estimation. Twenty mining stocks, out of the 801 listed in the ASX as of December 2012, have been selected for the analysis under the criteria of satisfying the eight years trading period sought, having very weak or no autocorrelation of residuals and displaying the highest kurtosis. Models' estimations of dependence are compared and inserted into a differential evolution algorithm for non-convex global optimization in order to conduct risk controlled CVaR portfolio optimization (Ardia, Boudt, Carl, Mullen \& Peterson, 2011) and be able to identify the one yielding the highest portfolio return. The findings are of relevance in portfolio allocation and portfolio risk management.

Energy and mining stock markets are subjected to numerous price drivers holding complex relationships. The dynamics of production and consumption based on seasonality features, transportation and storage, weather conditions, commodity price fluctuations, currency changes, market confidence and expectations, trading speculations and the domestic and international states of the economy impact mining stock prices in particular and unobvious ways reflected in high volatility with sudden spikes in the stock's return distribution (Pilipovic, 1998). The generation of accurate measurements of the dependence matrix of mining stock's return series is therefore both, a non-trivial task due to the hard to decipher characteristics present in return series suffering from high levels of kurtosis (Carvalho, Lopes \& Aguilar, 2010) and, a crucial element in portfolio optimization and portfolio risk management.
\end{abstract}

The use of graphical techniques in this study is justified on the basis of their utility and suitableness. Graphical models such as pair c-vine copulas, the graphical lasso and adaptive graphical lasso provide, for instance, the visualization and flexibility to represent a problem in a more simplified and dissected form (Lauritzen, 1996). Graphs also appear to be naturally adequate to express the interaction of variables and thus facilitate the analysis of their dependency. The models of dependence estimation and CVaR portfolio optimization, on the other hand, are desirable due to mathematical and statistical framework they provide which may lead to satisfactory results and, their apparent ability to overcome the flaws (i.e. standardized model application to all joint distributions, restrictive and deterministic linear and monotonic modelling functions as in the Pearson and Spearman) traditional measures display when dealing with highly kurtotic data, joint distributions with stronger dependence in the tails and controlled risk non-convex portfolio optimization problems. Findings indicate that the highest portfolio returns are generated by inserting the covariance output matrix from the student-t copula into the differential optimization algorithm and, the student-t copula fitting with separate modelling of the marginal distributions appears to be the most desirable modelling choice. The portfolio return by the adaptive graphical lasso is lower than that of the student-t and is followed by the Gaussian pair c-vine copula. The regular graphical lasso produced the lowest portfolio return and the covariance matrix values were higher for models producing the highest portfolio returns implying that the models generating the lowest portfolio returns underestimated the dependence of the assets. The implications of the findings suggest that specific modelling of each marginal distribution, as compared to modelling based on a Gaussian framework, may lead to an edge in the estimations due to the distribution differences encountered on each marginal. Furthermore, the ability of the model to capture dependence in the tails, as it is the case of the student- $t$ copula, does provide a modelling advantage too. This paper appears to be the first one in, comparing the portfolio performance of the models of dependence estimation in the context of controlled CVaR, applying the models treated to a highly kurtotic mining sample of stocks from the Australian market and modelling separately the distribution of the marginals when fitting the student- $t$ copula.

Keywords: Dependence structure, copula, CVaR, differential evolution, mining stocks. 
Arreola et al., Dependence estimation and controlled CVaR portfolio optimization of a highly kurtotic Australian mining sample of stocks.

\section{INTRODUCTION}

Dependence estimation techniques such as copula and pair copula modelling have, since Sklar's (1959) work, been adopted and developed fundamentally due to the possibility to benefit from the already identified and parameterized probability distributions of random variables. On the other hand, the regular and adaptive graphical least absolute shrinkage and selection operators (hereafter regular and adaptive graphical lassos) for sparsity generation and precision matrix (i.e. inverse covariance matrix) estimation has grown in popularity due to its model selection procedure which is based on the optimization of graphical structures; the fact that it helps reduce the model's estimation error (often expressed as an error term in regression models) and bias introduced by irrelevant-inactive variables (Tibshirani, Bien, Friedman, Hastie, Simon. Taylor \& Tibshirani J., 2012), the understanding that the optimal solution to many real world optimization problems is actually sparse and its dimension reduction attributes which permit the solving of large, and as a result, complex optimization problems we could only dream of solving in the past.

A bivariate copula is a statistical tool for the measurement of dependence in joint distributions. Its analytical structure is designed to split the marginal distribution from the dependence (i.e. copula) while still preserving the distribution of the marginal (Patton, 2012). Two well-known categories exist in the repertoire of bivariate copulas, the elliptical (e.g. Gaussian and student-t) and the Archimedean (e.g. Gumbel, Frank and Clayton). In stock's return series modelling, elliptical copulas tend to be inadequate when high kurtosis and asymmetric dependence exist. Archimedean copulas, on the other hand, although better than the elliptical under those circumstances, do not perform well in high dimensions due to the standardized application of only one or two parameters to the joint distribution of every pair of variables. From both categories, the student-t copula has been found to be the best performer (Demarta \& McNeil, 2005) in many financial applications.

Pair vine copulas, unlike the standard bivariate elliptical and Archimedean, offer the flexibility to design high dimensional distribution structures with specific-specialized bivariate copula modelling of joint distributions located at the nodes of the vine. Bivariate copula selection at the nodes is determined by the specific characteristics of the joint distribution being modelled (Brechmann \& Schepsmeier, 2011).

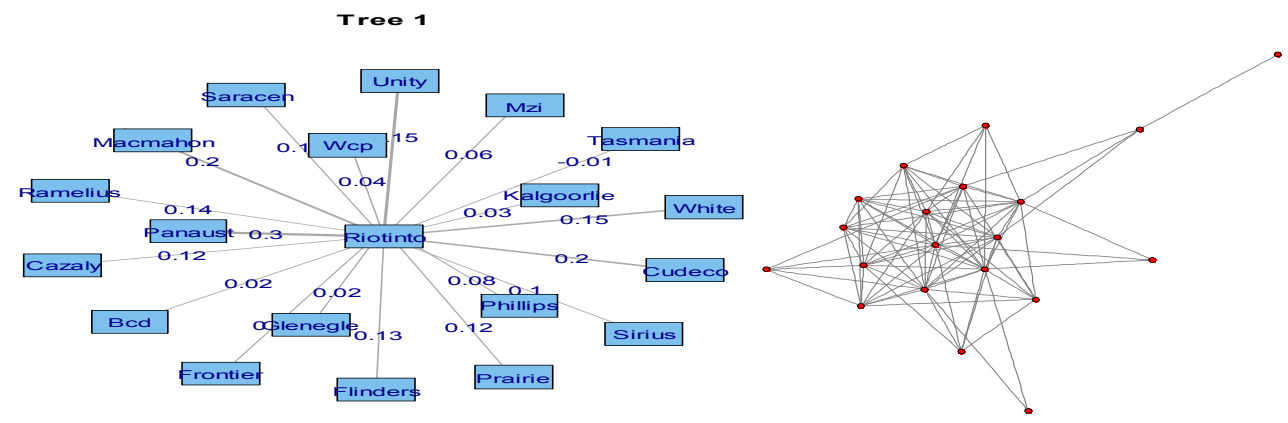

Figure 1: On the left, the first tree of applied Gaussian pair c-vine copula. On the right, the optimal graphical lasso structure of the precision matrix.

The regular and adaptive graphical lasso techniques deal with single equation objective function (e.g. regression models) and system of equations objective function (e.g. precision matrix estimation models) optimization problems through scalar and matrix weighted $\ell_{1}$-norm penalizations, respectively, with the purpose of achieving sparse optimal solutions (Fan, Feng \& Wu, 2009).

Research into the fields of dependence estimation and portfolio optimization has since the seminal works of Sklar (1959), Dempster (1972) and Markowitz (1952) attracted significant attention in the scientific community and financial sector. In the line of Sklar's theorem, the most prominent developments in dependence estimation have come in the form of copulas and pair vine copula constructions. Embrechts, McNeil and Straumann (1999) are the first to associate the concept of copula with measures of dependence within the financial arena and Junker and May (2005) are among the first who applied copulas to model portfolios of stocks with high risk. Concerning pair vine copulas, Brechmann and Schepsmeier (2011) point to the work of Joe (1997) as the first to deal with pair vine copula constructions. Subsequent significant developments on the subject are undertaken by Bedford and Cooke (2001) who for the first time derived, analytically, an equation providing a frame for multivariate probability distributions based on pair copulas. Aas, Czado, Frigessi and Bakken (2009) introduced the well-known analytical models used for the decomposition of densities and inference of canonical and drawable pair vine copulas. On the other hand, the Covariance Selection work of Dempster (1972) paved the way for the development of Tibshirani's (1996) regular graphical lasso for sparsity search and inverse covariance matrix estimation. In his work Tibshirani applies a greedy stepwise forward-backward method aimed at finding the smallest number of neighbours in a graph's nodes. Issues of stability for high penalty values and the need for more sparsity in large optimization problems would later on lead to Fan and Li's (2001) and Zou's (2006) improved versions of the regular 
Arreola et al., Dependence estimation and controlled CVaR portfolio optimization of a highly kurtotic Australian mining sample of stocks.

graphical lasso where both, model selection and parameter estimation are conducted simultaneously and a matrix weighted penalization (i.e. in the form of an adaptive graphical lasso) of the precision matrix components is applied, respectively. Fan, Feng and $\mathrm{Wu}$ (2009) found the adaptive graphical lasso to outperform the regular graphical lasso. Finally, while research into a mix of marginal risk control and CVaR portfolio optimization has been carried out by Boudt, Carl, and Peterson (2010), the differential evolution algorithm has been applied by Krink and Paterlini (2011) to optimize non-convex portfolios of stocks.

In this study we are strongly motivated to find out about the differences in dependence estimation and CVaR portfolio optimization arising from the implementation of the various modeling techniques suggested. Specifically, it is attempted to single out the model of dependence estimation generating the highest portfolio return. The motivation behind the selection of the specific data sample has to do with its anomalous statistical features and the eight years size of the sample aims to reduce the sampling error while also covering the preGFC, GFC and post-GFC. Traditional methods of dependence estimation would not be expected to adequately account for high levels of kurtosis in the return series and the models suggested are expected to do better.

\section{MODELS}

\subsection{Pair c-vine and student-t copulas}

Let $\boldsymbol{x}=\left(x_{1}, \ldots, x_{n}\right)$ be a sequence of random variables with sequences of continuous distribution and inverse distribution functions $F_{1}\left(x_{1}\right), \ldots, F_{n}\left(x_{n}\right)$ and $F_{1}^{-1}\left(x_{1}\right), \ldots, F_{n}^{-1}\left(x_{n}\right)$, respectively. Let, in addition to that, their corresponding probability density functions be $f_{1}\left(x_{1}\right), \ldots, f_{n}\left(x_{n}\right)$ and $f_{1}^{-1}\left(x_{1}\right), \ldots, f_{n}^{-1}\left(x_{n}\right)$. It then follows, their joint cumulative distribution and joint probability density functions are $F(\boldsymbol{x})=F\left(x_{1}, \ldots, x_{n}\right)$ and $f(\boldsymbol{x})=f\left(x_{1}, \ldots, x_{n}\right)$ and, according to a probability integral transform, a random variable $U_{i} \equiv F_{i}\left(X_{i}\right)$ is uniformly distributed on $[0,1]$ and has a reverse expression $X_{i}=F_{i}^{-1}\left(U_{i}\right)$, for $i=1, \ldots, n$. Applying the aforementioned distributional relationship between $U_{i}$ and $X_{i}$, the joint probability distribution of $U$ can be written as follows:

$$
\begin{aligned}
P\left(X_{1} \leq F_{1}^{-1}\left(x_{1}\right), \ldots, X_{n} \leq F_{n}^{-1}\left(x_{n}\right)\right) & =\mathrm{P}\left(U_{1} \leq x_{1}, \ldots, U_{n} \leq x_{n}\right) \\
& \equiv \mathrm{C}\left(x_{1}, \ldots x_{n}\right)
\end{aligned}
$$

Making use of the property of inverse distributions $F_{i}\left(F_{i}^{-1}\left(U_{i}\right)\right) \geq U_{i}$ and dividing the first term of the inequalities on both sides of (1) by $F_{i}$ it can be shown that,

$$
\begin{aligned}
P\left(X_{1} \leq x_{1}, \ldots, X_{n} \leq x_{n}\right) & =\mathrm{P}\left(F_{1}\left(X_{1}\right) \leq F_{1}\left(x_{1}\right), \ldots, F_{n}\left(X_{n}\right) \leq F_{n}\left(x_{n}\right)\right) \\
& =\mathrm{C}\left(F_{1}\left(x_{1}\right), \ldots F_{n}\left(x_{n}\right)\right)
\end{aligned}
$$

Then as a result of Sklar's theorem (Sklar, 1959), the following equivalent expression of (2) should hold,

$$
F(\boldsymbol{x})=F\left(x_{1}, \ldots, x_{n}\right)=\mathrm{C}\left(F_{1}\left(x_{1}\right), \ldots F_{n}\left(x_{n}\right)\right)
$$

$\mathrm{C}$ in (1), (2) and (3) is a copula representing the joint distribution function of the $\mathrm{n}$-dimensional random variable on $[0,1]^{n}$ with uniform margins $F_{1}, \ldots, F_{n}$. In the application of the Gaussian pair canonical vine copula we use the following analytical model suggested by Aas et al.,(2009) for the decomposition of the multivariate probability density:

$f(\boldsymbol{x})=f\left(x_{1}, \ldots, x_{n}\right)=\prod_{k=1}^{n} f_{k}\left(x_{k}\right) \cdot \prod_{i=1}^{n-1} \prod_{j=1}^{n-i} c_{i, i+j \mid 1:(i-1)}\left(\mathrm{F}\left(x_{i} \mid x_{1}, \ldots x_{i-1}\right), \mathrm{F}\left(x_{i+j} \mid x_{1}, \ldots, x_{i-1}\right) \mid \boldsymbol{\theta}_{i, i+j \mid 1:(i-1)}\right)$

An example of 4-dimensional c-vine density decomposition and graph are:

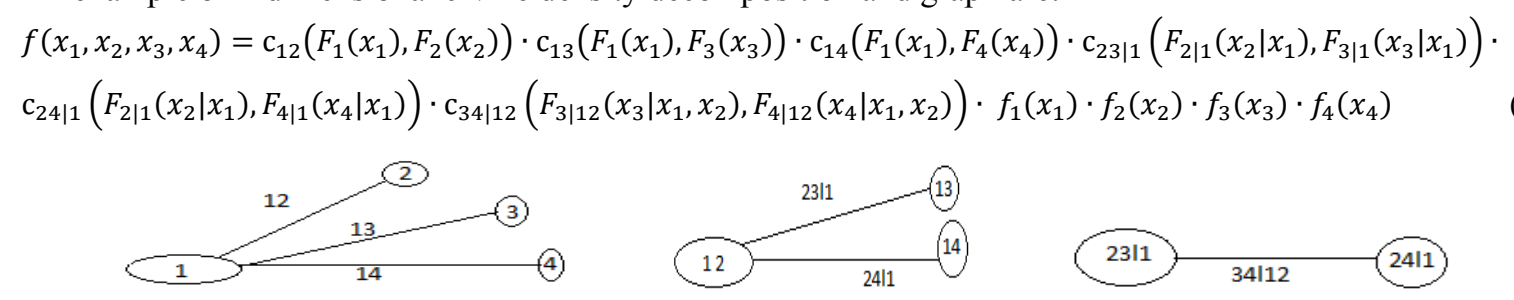

Figure 2: A four dimensional pair c-vine graph

The student-t copula equation is:

$$
C_{\rho, v}(u, v)=\int_{-\infty}^{t_{v}{ }^{-1}(u)} \int_{-\infty}^{t_{v}{ }^{-1}(v)} \frac{1}{2 \pi\left(1-\rho^{2}\right)^{1 / 2}}\left\{1+\frac{x^{2}-2 \rho x y+y^{2}}{v\left(1-\rho^{2}\right)}\right\}^{-\frac{v+2}{2}} d x d y
$$


Arreola et al., Dependence estimation and controlled CVaR portfolio optimization of a highly kurtotic Australian mining sample of stocks.

Here, $\rho$ is the dependence coefficient, $v$ the degrees of freedom parameter and $t_{v}{ }^{-1}(\cdot)$ the inverse distribution with mean 0 and variance $\frac{v}{v-2}$.

\subsection{Regular and adaptive graphical lasso}

Estimation of the sparse dependence matrix through the regular and adaptive graphical lasso is accomplished by solving the following optimization problems:

$$
\begin{aligned}
& \log \operatorname{det} \Omega-\operatorname{tr}(\Omega \boldsymbol{S})-\lambda \sum_{i=1}^{n} \sum_{i=1}^{n}\left|w_{i j}\right| \\
& \log \operatorname{det} \Omega-\operatorname{tr}(\Omega \boldsymbol{S})-\lambda \sum_{i=1}^{n} \cdot \sum_{j=1}^{n} \varsigma_{i, j}\left|\omega_{i, j}\right|
\end{aligned}
$$

Here, $\boldsymbol{S}$ is the sample covariance matrix, $\Omega$ the precision matrix, $w_{i j}$ the matrix componenets of $\Omega, \lambda$ the penalizing or tuning parameter, det refers to the determinant, $\operatorname{tr}$ refers to the trace and $\varsigma_{i, j}=\frac{1}{\left|\omega_{i, j}\right|^{\gamma}}$ in (8) is the penalty matrix of weights applied to the precision matrix components in the adaptive graphical lasso problem (Fan et al., 2009).

\subsection{Differential evolution CVaR portfolio optimization}

The method uses floating-point encoding of a population, arithmetic operators and alteration for the selection and evolution of potential solutions and converges systematically by applying transformations (i.e. differential mutations) on vectors of parameters identified as the population. After every differential parameter vectors with potential to solve the minimization problem are kept to undergo a subsequent transformation and so on until a global optimal solution is encountered. Analytically, let $x_{i, g}$ represent an existing population with $i$ vectors of parameters and $g$ generations. The first transformed or mutated vector of parameters $v_{i, g}$ is generated by randomly selecting three population members $x_{r 0, g}, x_{r 1, g}$ and $x_{r 2, g}$ or,

$$
v_{i, g}=x_{r 0, g}+F \cdot\left(x_{r 1, g}-x_{r 2, g}\right)
$$

where $F$ is a scaling factor with values greater than zero and less than one (Ardia et al., 2011). Controlling the risk contribution percentage of individual stocks in a portfolio is an idea first proposed in Boudt et al., (2010). According to them the percentage CVaR contribution of asset $i$ is represented by the function:

$$
\frac{w_{i}\left[-\mu_{i}+\frac{\left(\sum w\right)_{i}}{\sqrt{w^{\prime} \sum w}} \frac{\phi\left(z_{\alpha}\right)}{\alpha}\right]}{w^{\prime} \mu+\sqrt{w^{\prime} \sum w} \frac{\phi\left(z_{\alpha}\right)}{\alpha}}
$$

Here $\boldsymbol{w}=\left(w_{1}, \ldots w_{d}\right)^{\prime}$ is a vector of weights with mean $\boldsymbol{\mu}=\left(\mu_{1}, \ldots \mu_{d}\right)^{\prime}$ and covariance matrix $\sum$.

\section{DATA AND MODEL APPLICATION}

The quantitative analysis is based on a highly kurtotic mining sample of stocks from the Australian market with daily price frequency.
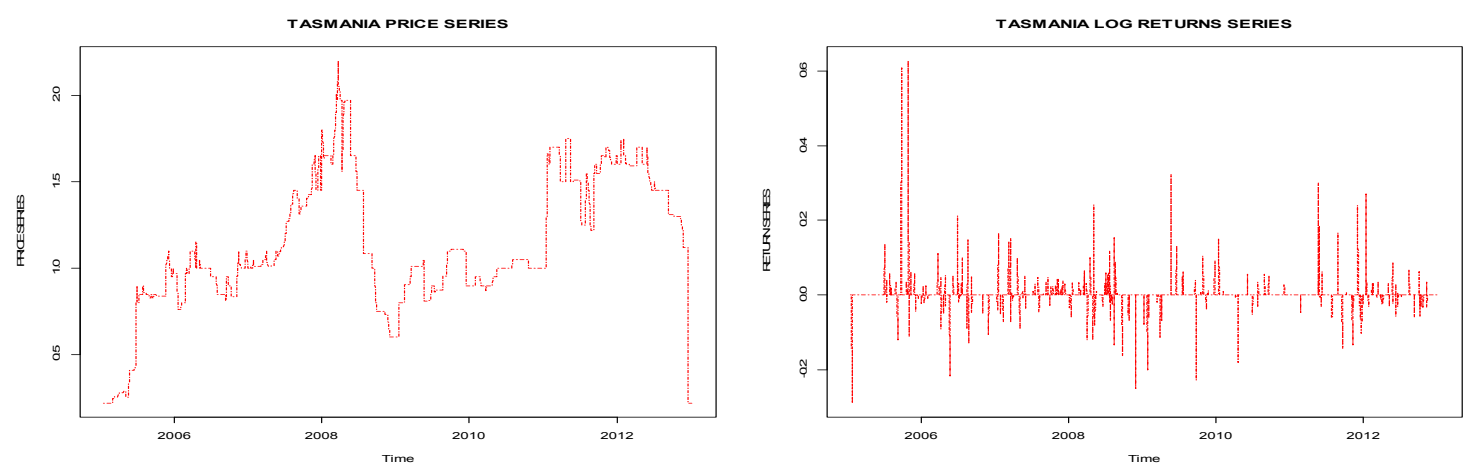

Figure 3: Price and log return series of Tasmania mining stock

Logarithmic returns are estimated and the time series' length stretches from January 7, 2005 to January 4, 2013. The specific selection of eight years price series is aimed at reducing the sampling error and covers the pre-GFC, GFC and post-GFC. All 20 stock return series have very weak or no autocorrelation of residuals. The stocks names can be found in table 1 of appendix A. The return series have been filtered with an ARMA $(1,1)-G A R C H(1,1)$ with student-t innovations and, a probability integral transform has been applied to the 
Arreola et al., Dependence estimation and controlled CVaR portfolio optimization of a highly kurtotic Australian mining sample of stocks.

standardized residuals in order to obtain the copula data. In the application of the Gaussian pair c-vine copula, the column order of the stock return series in the data set has been arranged according to the criterion suggested by Czado, Schepsmeier and Min (2012). Rio Tinto stock's return series takes the first column because it has the strongest dependence with the other variables and so on with the rest following the order from left to right of row 1 in table 1 of appendix A.

\subsection{Fitting of models and output values}

In order to estimate the dependence of the variables through the student-t copula fitting, we have adopted two approaches: first we have let the R package "copula" algorithm identify the distribution of the marginals and corresponding parameters and, compute dependence of the variables; second, we have fitted separately ten of the most common distribution functions to every marginal with the aim of identifying their distribution and corresponding parameters, then, the output was fed into the aforementioned student-t copula algorithm. Figure 4 depicts the fitting of ten distribution functions to Cazaly's return distribution.
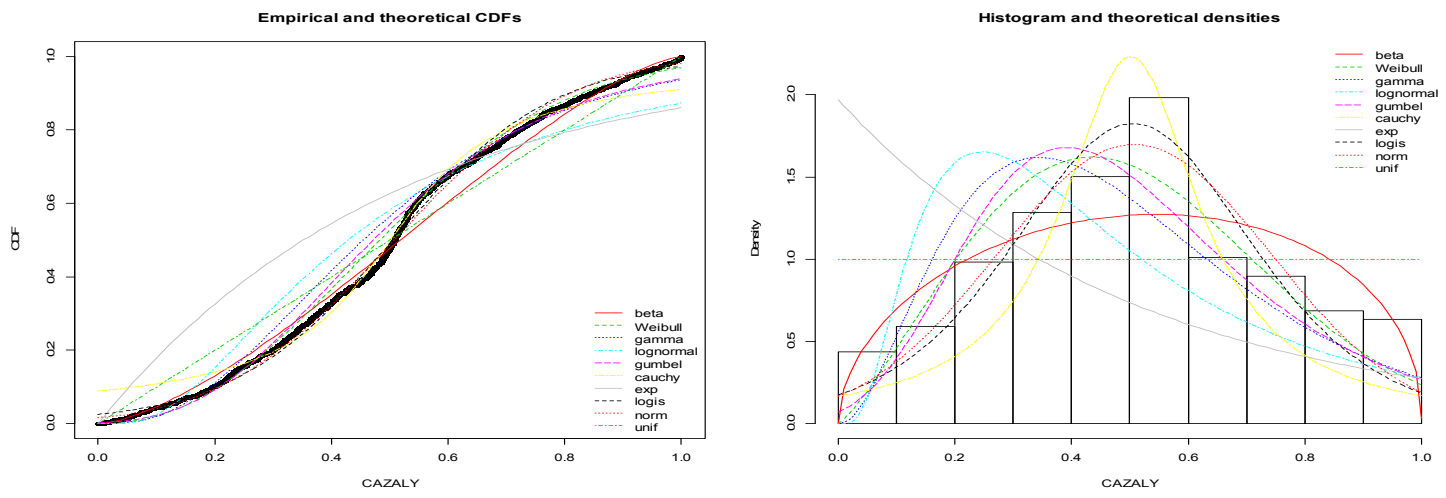

Figure 4: Multiple distribution fitting to Cazaly mining stock

According to both plots and the returned Akaike and Bayesian information criteria values, the probability distribution best describing the behaviour of Cazaly's return distribution is the beta function. The existence of visible fat tails is probably the reason behind the selection of the beta distribution function. The probability distribution functions best fitting the stocks return distributions are: Riotinto (beta), Panaust (beta), Cudeco (beta), Unity (beta), Mcmahon (beta), Saracen (normal), Praire (normal), Cazaly (beta), Ramelius (beta), White (normal), Wcp (cauchy), Kalgoorlie (exponential), Bcd (logistic), Philips (logistic), Flinders (beta), Frontier (logistic), Sirius (logistic), Tasmania (logistic), Gleneagle (logistic) and Mzi (cauchy) .

In the computation of controlled risk portfolio optimization we have used the model's estimation of dependence as input parameters in the differential evolution algorithm. The following analysis has been conducted on all portfolio optimizations. Resulting values can be found in table lof appendix A.

We start by computing the risk contribution of every stock to the overall risk of the portfolio using an equal $5 \%$ investment on each of the twenty stocks. Knowing the risk contribution is important because it tells of the proportionality in the weight to risk contribution ratio. Also, at this point we do not care about controlling the down side risk of the portfolio. Next, once having obtained information about the risk contribution of every stock to the risk of the portfolio, we impose a fixed 3\% risk contribution constraint on every stock in the portfolio and optimize it. The generated outputs are the weights on every stock of the CVaR or minimum risk portfolio and the risk of the portfolio. Finally, we let the risk contribution float (i.e. impose no constraint on it) and optimize the portfolio targeting the highest possible diversification of risk contribution (i.e. minimizing the biggest $\mathrm{CVaR}$ contribution). This problem is non-convex and can be easily solved via the differential evolution method. For practical purposes, in table 1 of appendix A, only one decimal has been considered in the weights and we have set the abbreviations ( $\mathrm{rc}$ ) to mean the risk contribution, (smm rc) the mean separate marginal modelling risk contribution, (mrpw) the minimum risk portfolio weights, (smm mrpw) the separate marginal modelling minimum risk portfolio weights, (maxrcd) the maximum risk diversification and (smm maxrcd) the separate marginal modelling maximum risk contribution diversification.

Looking at table 1 in appendix A, according to the student-t copula modelling $10.85 \%$ of the portfolio conditional value at risk is generated from investing $5 \%$ in Rio Tinto. When a $3 \%$ risk contribution constraint was set the optimal amount to invest in Rio Tinto is $2.6 \%$ and the return of the portfolio is $26.41 \%$ with a -0.011 portfolio risk. On the other hand, when letting the risk contribution to float the optimal amount to invest in Rio Tinto is $2.3 \%$. On the other hand, the portfolio return with a 3\% risk contribution constraint when using the dependence estimation computed through the student-t copula with separate marginal modelling is, $27.11 \%$ with portfolio risk -0.015 . The portfolio return with a $3 \%$ risk contribution constraint 
Arreola et al., Dependence estimation and controlled CVaR portfolio optimization of a highly kurtotic Australian mining sample of stocks.

when using the dependence estimation computed through the Gaussian pair c-vine copula is $24.67 \%$ with a portfolio risk of -0.01 . The regular graphical lasso generated a $24.58 \%$ portfolio return with a -0.012 portfolio risk and the adaptive graphical lasso a $25.98 \%$ portfolio return with a portfolio risk of -0.011 .

\section{CONCLUSION}

The numbers indicate that the highest portfolio returns are generated by inserting the covariance output matrix from the student- $t$ copula into the differential optimization algorithm. Moreover, the student-t copula along with separate modelling of the marginal distributions appears to be the most desirable modelling choice out of the models applied in this study. The portfolio return with the adaptive graphical lasso covariance matrix was higher as compared to the Gaussian pair C-vine copulas. The lowest portfolio return was produced when the covariance matrix of regular graphical lasso was utilized. The numbers in the covariance matrices from all models were higher for higher portfolio returns. It could be inferred then that models generating the lowest portfolio returns underestimated the underlying dependence of the assets. It could be concluded then that the student-t copula is the most adequate modelling tool in dealing with the highly kurtotic data suggested and, the results add to the reputation the student-t copula has gained in the financial arena. Finally, the student-t copula is suggested as a good modelling tool in the estimation of dependence of highly kurtotic mining stocks.

\section{REFERENCES}

Aas, K., Czado, C., Frigessi, A. and Bakken, H. (2009). Pair-copula constructions of multiple dependence. Insurance: Mathematics and Economics Journal, 44(2), 182-198.

Ardia, D., Boudt, K., Carl, P., Mullen, K. and Peterson, B. (2011). Differential evolution with DEoptim: an application to non-convex portfolio optimization. The $R$ Journal, 3(1), 27-34.

Ardia, D., Mullen, K., Ulrich J. and Peterson, B. (2011). DEoptim: an R package for global optimization by differential evolution. Journal of Statistical Software, 40(6), 1-26.

Bedford, T. and Cooke, R.M. (2001). Probability density decomposition for conditionally dependent random variables modelled by vines. Annals of Mathematics and Artificial intelligence, 32, 245-268.

Boudt, K., Carl, P. and Peterson B. G. (2010). Portfolio optimization with conditional value-at-risk budgets.

Brechmann, E.C. and Schepsmeier, U. (2011). Modelling dependence with c and d vine copulas: The Rpackage C-D vine. Journal of Statistical Software, 52(3), 1-26.

Carvalho, C. M., Lopes, H. F. and Aguilar O. (2010). Dynamic stock selection strategies: a structured factor model framework. Bayesian Statistics, 9.

Czado, C. Schepsmeier, U. \& Min, A. (2012). Maximum likelihood estimation of mixed c-vines with application to exchange rates. Statistical Modelling, 12(3), 229-255.

Dempster, A. (1972). Covariance selection. Biometrics, 157-175.

Demarta, S., and McNeil, A. J. (2005). The t copula and related copulas. International Statistical Review, 73( 1) 111-129.

Embrechts, P., McNeil, A. and Straumann, D. (1999). Correlation: pitfalls and alternatives. Risk, 12(5), 6971.

Fan, J. and Li, R. (2001). Variable selection via nonconcave penalized likelihood and its oracle properties. Journal of American Statistical Association. 96(456), 1348-1360.

Fan, J., Feng, Y. and Wu Y. (2009). Network exploration via the adaptive lasso and scad penalties. The Annals of Applied Statistics, 3(2), 521-541.

Joe, H. (1997). Multivariate Models and Dependence Concepts. Chapman and Hall, London.

Junker, M., and May, A. (2005). Measurement of aggregate risk with copulas. The Econometrics Journal. 8, $428-454$.

Krink, T. and Paterlini, S. (2011). Multiobjective optimization using differential evolution for real- world portfolio optimization. Computational Management Science, 8,157-179.

Lauritzen, S.L. (1996). Graphical Models. Oxford University Press, Oxford.

Markowitz, H. M. (1952). Portfolio selection. Journal of Finance, 7, 77-91.

Patton, A.J. (2012). A review of copula models for economic time series. Journal of Multivariate Analysis, 110, 4-18.

Pilipovic, D. (1998). Energy risk: valuing and managing energy derivatives. New York, McGraw-Hill. 
Arreola et al., Dependence estimation and controlled CVaR portfolio optimization of a highly kurtotic Australian mining sample of stocks.

Sklar, A. (1959). Functions de repartition and dimensions et leurs marges. l'Institut de Statistique de L'Universit'e de Paris, 8, 229-231.

Tibshirani, R. (1996). Regression shrinkage and selection via the LASSO. Journal of the Royal Statistical Society, 58(1), 267-288.

Tibshirani, R., Bien, J., Friedman, J., Hastie, T., Simon., Taylor J., and Tibshirani R.J. (2012). Strong rules for discarding predictors in lasso-type problems. Journal of the Royal Statistical Society, 74(2), 245-266.

Zou, H. (2006). The adaptive lasso and its oracle properties. Journal of American Statistical Association, $10(1), 1418-1429$.

\section{APPENDIX A}

Table 1. Differential evolution optimization weights with models' estimations of dependence as inputs

\begin{tabular}{|c|c|c|c|c|c|c|c|c|c|c|c|c|c|c|c|c|c|c|c|c|}
\hline 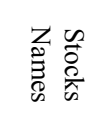 & 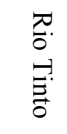 & 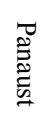 & $\begin{array}{l}\Omega \\
\overparen{2} \\
\check{0}\end{array}$ & $\underset{\gtrless}{\stackrel{\Xi}{\rightleftarrows}}$ & 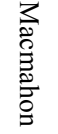 & 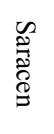 & T. & $\begin{array}{l}\text { N̂} \\
\stackrel{N}{<}\end{array}$ & 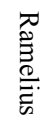 & 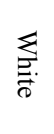 & 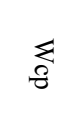 & 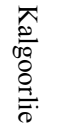 & $\stackrel{\varpi}{\check{D}}$ & 导 & 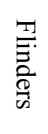 & $\begin{array}{l}T \\
\stackrel{0}{0} \\
\stackrel{0}{9}\end{array}$ & 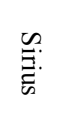 & 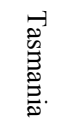 & 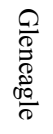 & s. \\
\hline \multicolumn{21}{|c|}{ Differential evolution weights with student-t copula's dependence matrix as input } \\
\hline $\mathrm{rc}$ & 10.8 & 9.9 & 6.8 & 7.1 & 7.3 & 6 & 4.4 & 4.5 & 6.7 & 4.9 & 3.2 & 3.1 & 3.9 & 2.5 & 5.4 & 2.3 & 3 & .59 & 3.8 & 2.7 \\
\hline smm rc & 11 & 9.3 & 6.7 & 7.5 & 7.6 & 6 & 4.4 & 4.6 & 6.9 & 4.6 & 3.2 & 3.4 & 4 & 2.5 & 5.4 & 2.3 & 3 & .63 & 3.3 & 2.8 \\
\hline mrpw & 2.6 & 2.8 & 4 & 3.6 & 3.1 & 3.9 & 4.4 & 4.5 & 3.8 & 5 & 6 & 5.8 & 5.4 & 6.9 & 4.5 & 7.1 & 6.7 & 6.9 & 5.3 & 6.9 \\
\hline $\begin{array}{l}\text { smm } \\
\text { mrpw }\end{array}$ & 2.6 & 3 & 4.1 & 3.9 & 3.8 & 4.3 & 5.4 & 5.3 & 4.1 & 5.1 & 6.3 & 6.2 & 5.7 & 6.2 & 4.7 & 5.6 & 6.2 & 4.9 & 5.4 & 6.2 \\
\hline maxrcd & 2.3 & 3 & 3.7 & 3.8 & 4 & 4.5 & 5.4 & 4.9 & 3.8 & 5.2 & 6.1 & 6.1 & 5.8 & 7.6 & 4.8 & 7.4 & 2.6 & 7.7 & 5.1 & 5.2 \\
\hline $\begin{array}{c}\text { smm } \\
\text { maxrcd }\end{array}$ & 2.4 & 3 & 4.1 & 4 & 4.1 & 4.7 & 5.6 & 5.6 & 4.1 & 4.9 & 5.5 & 1.9 & 5.7 & 6.6 & 5.1 & 6.8 & 6.3 & 6.7 & 5.7 & 6.5 \\
\hline \multicolumn{21}{|c|}{ Differential evolution weights with Gaussian pair c-vine's dependence matrix as input } \\
\hline $\mathrm{rc}$ & 12.9 & 9.1 & 5.7 & 7.0 & 5.9 & 4.9 & 4.6 & 5.0 & 5.7 & 5.3 & 3.2 & 3.2 & 3.2 & 3.1 & 5.5 & 2.9 & 3.4 & 1.0 & 3.4 & 3.8 \\
\hline mrpw & 2.3 & 3.2 & 4.1 & 3.6 & 3.9 & 4.9 & 4.7 & 4.7 & 4.4 & 4.4 & 5.7 & 6.0 & 6.0 & 6.5 & 4.3 & 6.9 & 6.0 & 6.6 & 5.6 & 5.4 \\
\hline maxrcd & 2.4 & 3.2 & 4.6 & 3.8 & 4.7 & 4.4 & 5.1 & 4.8 & 4.7 & 4.4 & 5.9 & 5.1 & 6.2 & 6.7 & 4.4 & 7.2 & 6.3 & 7.4 & 2.3 & 5.7 \\
\hline \multicolumn{21}{|c|}{ Differential evolution weights with regular graphical lasso's dependence matrix as input } \\
\hline $\mathrm{rc}$ & 9.1 & 8.4 & 6.7 & 6.4 & 6.8 & 6.0 & 5.1 & 4.9 & 5.9 & 5.6 & 3.2 & 2.8 & 3.6 & 4.5 & 5.3 & 4.2 & 3.2 & 0.9 & 3.3 & 3.4 \\
\hline mrpw & 3.0 & 3.3 & 3.7 & 3.7 & 4.1 & 4.4 & 4.5 & 4.9 & 4.5 & 4.5 & 6.1 & 6.4 & 5.8 & 5.0 & 4.8 & 5.5 & 6.2 & 6.4 & 6.1 & 6.1 \\
\hline maxrcd & 3.3 & 3.6 & 4.4 & 4.5 & 4.2 & 4.3 & 5.3 & 4.6 & 4.8 & 4.5 & 6.5 & 0.6 & 6.3 & 5.7 & 5.0 & 5.7 & 6.4 & 6.5 & 6.4 & 6.5 \\
\hline \multicolumn{21}{|c|}{ Differential evolution weights with adaptive graphical lasso's dependence matrix as input } \\
\hline $\mathrm{rc}$ & 9.8 & 8.7 & 6.9 & 6.4 & 7.0 & 6.1 & 4.9 & 4.7 & 6.0 & 5.6 & 3.1 & 2.6 & 3.3 & 4.2 & 5.2 & 4.0 & 3.2 & 1.0 & 3.2 & 3.1 \\
\hline mrpw & 2.8 & 3.0 & 3.6 & 4.1 & 3.6 & 4.4 & 4.9 & 5.0 & 4.4 & 4.7 & 6.2 & 6.5 & 6.0 & 5.4 & 4.9 & 5.4 & 6.1 & 6.6 & 5.5 & 6.0 \\
\hline maxrcd & 2.9 & 3.6 & 4.3 & 3.9 & 4.2 & 4.7 & 4.5 & 4.8 & 4.4 & 5.0 & 6.4 & 6.8 & 6.2 & 5.8 & 4.9 & 5.5 & 4.7 & 7.7 & 2.4 & 6.6 \\
\hline
\end{tabular}

